Proc. Estonian Acad. Sci. Phys. Math., 1989, 38, N 4, '460-462

удК 681.511 .42

Olle KOTTA

\title{
APPROXIMATE INPUT-OUTPUT LINEARIZATION OF DISCRETE-TIME NONLINEAR SYSTEMS
}

WOlle KOTTA, DISKREETSE MITTELINEAARSE SUSTEEMI SISEND-VALJUND KUJUTISE LIGI, IKAUDNE LINEARISEERIMINE

ЖОЛЛЕ КОТТА. ПРИБЛИЖЕННАЯ ЛИНЕАРИЗАЦИЯ ВХОД-ВЫХОДНОГО ОТОБРАЖЕНИЯ НЕЛИНЕИНОИ СИСТЕМЫ ДИСКРЕТНОГО ВРЕМЕНИ

\section{(Presented by 0. Jaaksoo)}

\section{Introduction}

Many researchers have tackled the input-output linearization problem as finding a static state feedback such that the input-output maps (inputdependent part) of the resulting closed-loop system and some linear system coincide. For discrete-time systems this problem has been considered in $\left[{ }^{1-3}\right]$. Generally, the linearizing feedback is not linear in new control, and in $\left[{ }^{1,3}\right]$ it has been found in the form of formal power series. In practical situations, of course, one must confine oneself to some finite terms in power series. The question therefore arises: how much does the use of this approximate solution, instead of the exact solution, influence the desired result. It will be shown in this paper that if we take into consideration $k$ terms in this series, then the Volterra kernels of the closedloop systems up to the $k$ th order will coincide for an approximate and an exact solution.

\section{Exact input-output linearization.}

Consider the nonlinear discrete-time system described by equations

$$
\begin{gathered}
x(t+1)=x(t)+f_{0}(x(t))+\sum_{i \geqslant 1} f_{i}(x(t)) u^{i}(t), x(0)=x_{0}, \\
y(t)=h(x(t)),
\end{gathered}
$$

where the state $x(t) \in R^{n}$, the input $u(t) \in R$, the output $y(t) \in R$, $f_{i}: R^{n} \rightarrow R^{n}, i \geqslant 0$ and $h: R^{n} \rightarrow R$ are analytic functions on $R^{n}, f_{0}\left(x_{0}\right)=0$.

With reference to analytic functions $f(x): R^{n} \rightarrow R^{n}$ and $g(x): R^{n} \rightarrow R^{n}$, the following differential operators can be introduced $[1,2]$ :

$$
\begin{aligned}
L_{f}^{\otimes 0} & =1, \\
L_{f}^{\otimes r} & =\sum_{i_{s}, \ldots, i_{r}=1}^{n} f_{i_{1}} \ldots f_{i_{r}} \frac{\partial^{r}}{\partial x_{i_{1}} \ldots \partial x_{i_{r}}}, r \geqslant 1, \\
\Delta_{f} & =\sum_{k \geqslant 0} \frac{1}{k !} L_{f}^{\otimes k}, \\
L_{f}^{\otimes r} \otimes L_{g}^{\otimes s} & =\sum_{i_{1}, \ldots, j_{s}=1}^{n} f_{i_{1}} \ldots f_{i_{r}} g_{j_{s}} \ldots g_{j_{s}} \frac{\partial^{r+s}}{\partial x_{i_{1}} \ldots \partial x_{j_{s}}},
\end{aligned}
$$

where $f_{i}, g_{j}, i, j=1, \ldots, n$, and $I$ denote the ith component of $f, j$ th component of $g$ and the identity operator, respectively.

Let us introduce the differential operators associated with the system (1) $-(2)$ :

$\delta_{s}=\sum \frac{1}{n_{1} ! \ldots n_{k} ! \ldots} \Delta_{f_{0}} \otimes L_{f_{1}}^{\otimes n_{1}} \otimes \ldots \otimes L_{f_{k}}^{\otimes n_{k}} \otimes \ldots, \quad s=0,1,2 \ldots$, 
where the summation is taken over all sets of nonnegative integers $n_{i}$ such that the equation $n_{1}+2 n_{2}+\ldots+k n_{k}+\ldots=s$ holds.

Moreover, let

$$
a_{s}(x)=\left.\delta_{s} \circ \delta_{0}^{d} h(x)\right|_{x},
$$

where $d$ is the relative order of the system, i. e. $t=d+1$ is the first instant of time at which the output is affected by the control at $t=0$ $\left[{ }^{1,3}\right]$; by $《 \circ »$ is denoted the composition of operators, $\delta^{k}=\delta \circ \ldots \circ \delta$ ( $k$ - multiple composition) and

$$
\alpha_{k}(x)=\Sigma \frac{\left(k+c_{1}+\ldots+c_{k}-1\right) !}{k ! c_{2} ! \ldots c_{k} ! a_{1}^{k}(x)}\left(-\frac{a_{2}(x)}{a_{1}(x)}\right)^{c_{2}} \ldots\left(-\frac{a_{k}(x)}{a_{1}(x)}\right)^{c_{k}},
$$

where the summation is taken over all sets of nonnegative integers $c_{i}$ such that the equation $c_{2}+2 c_{3}+\ldots+(k-1) c_{k}=k-1$ holds. The following theorem holds. ${ }^{*}$

Theorem [1]. Consider the system (1), (2) for which $\delta_{1}^{\circ} \delta_{0}^{d}$ $\left.h(x)\right|_{x} \neq 0$ on some open and dense subset $V$ of $R^{n}$. Then the input/ /output linearization problem can be solved on $V$ by the following feed= back

$$
u(t)=\sum_{n \geqslant 1} \alpha_{n}(x(t))\left[v(t)-a_{0}(x(t))\right]^{n} .
$$

\section{Approximate linearization}

In this section we shall compare the Volterra kernels of two closed. loop systems, one of which is obtained by using the feedback (3) and the other by the feedback

$$
u^{*}(t)=\sum_{n=1}^{r} \alpha_{n}(x(t))\left[v(t)-a_{0}(x(t))\right]^{n},
$$

which takes into account only the first $r$ terms in the series (3). The equations of closed-loop systems we get by substituting one formal series into another and they are

and

$$
x(t+1)=x(t)+f_{0}(x(t))+\sum_{i \geqslant 1} \xi_{i}(x(t))\left[v(t)-a_{0}(x(t))\right]^{i}
$$

$$
x(t+1)=x(t)+f_{0}(x(t))+\sum_{i \geqslant 1} \gamma_{i}(x(t))\left[v(t)-a_{0}(x(t))\right]^{i},
$$

:respectively. Here [ $\left.{ }^{4}\right]$

$$
\xi_{i}=\sum_{l_{1}+2 l_{2}+\ldots+i l_{l}=i} \frac{\left|l_{1}+l_{2}+\ldots+l_{i}\right| !}{l_{1} ! \ldots l_{i} !} f_{\left|l_{1}+\ldots+l_{l}\right|} \alpha_{1}^{l_{1}} \ldots \alpha_{i}^{l_{l}}
$$

and

$$
\gamma_{i}=\left\{\begin{array}{l}
\xi_{i}, \text { if } i \leqslant r \\
\sum_{l_{1}+2 l_{2}+\ldots+r l_{r}=i} \frac{\left|l_{1}+l_{2}+\ldots+l_{r}\right| !}{l_{1} ! \ldots l_{r} !} f_{\left|l_{1}+\ldots+l_{r}\right|} \alpha_{1}^{l_{1}} \ldots \alpha_{r^{\prime}}^{l_{n}} \text { if } i>r,
\end{array}\right.
$$

The output equations for both systems coincide with equation (2).

Let us introduce the notations

* The result for linear analytic systems (i. e. for case $g_{i}(x)=0, i \geqslant 2$ ) can be obtained as the conclusion from this Theorem. Although the result of Monaco and Normand-Cyrot $\left[{ }^{3}\right]$ for such system is given in the different form, it can be shows by little manipulation that both coincide. 


$$
\begin{gathered}
\tilde{\delta}_{s}=\sum \frac{1}{n_{1} ! \ldots n_{k} ! \ldots} \Delta_{f_{0}} \otimes L_{\xi_{1}}^{\otimes n_{1}} \otimes \ldots \otimes L_{\xi_{k}}^{\otimes n_{k}} \otimes \ldots, \\
s=0,1,2, \ldots, \\
\delta_{s}^{*}=\sum \frac{1}{n_{1} ! \ldots n_{k} ! \ldots} \Delta_{f_{0}} \otimes L_{\gamma_{1}}^{\otimes n_{1}} \otimes \ldots \otimes L_{\gamma_{k}}^{\otimes n_{k}} \otimes \ldots, \\
s=0,1,2, \ldots,
\end{gathered}
$$

where in both cases the summation is taken over all sets of nonnegative integers $n_{i}$ such that the equation $n_{1}+2 n_{2}+\ldots+k n_{k}+\ldots=s$ holds.

The closed-loop systems (5), (2) and (6), (2) are analytic in state and polynomial in new control $v(t)$. The input-output behavior of these polynomial-analytic systems around the equilibrium point $v(t)=a_{0}(x(t))$ can be expressed by Volterra series $\left.{ }^{5}\right]$ :

$$
\begin{gathered}
y(t+1)=w_{0}(t+1)+\sum_{\tau_{1}=0}^{t} w_{1}\left(t+1, \tau_{1}\right)\left[v\left(\tau_{1}\right)-a_{0}\left(x\left(\tau_{1}\right)\right)\right]+\ldots \\
\ldots+\sum_{\tau_{1}=0}^{t} \sum_{\tau_{2}=\tau_{1}}^{t} \ldots \sum_{\tau_{k}=\tau_{k-1}}^{t} w_{k}\left(t+1, \tau_{1}, \ldots \tau_{k}\right) \times \\
\times\left[v\left(\tau_{1}\right)-a_{0}\left(x\left(\tau_{1}\right)\right)\right] \ldots\left[v\left(\tau_{k}\right)-a_{0}\left(x\left(\tau_{k}\right)\right)\right]+\ldots
\end{gathered}
$$

The kernels for the system (5), (2) are

$w_{0}(t+1)=\left.\tilde{\delta}_{0}^{t+1} h\right|_{x_{0}}$,

$w_{1}\left(t+1, \tau_{1}\right)=\left.\tilde{\delta}_{0}^{\tau_{\mathrm{i}} \circ} \tilde{\delta}_{1} \circ \tilde{\delta}_{0}^{t-\tau_{1} h}\right|_{x_{0}}$,

$w_{h}\left(t+1, \tau_{1}, \ldots, \tau_{k}\right)=\left.\tilde{\delta}_{0}^{\tau} \circ \tilde{\delta}_{1} \circ \tilde{\delta}_{1}^{\tau_{2}-\tau_{1}-1} \circ \tilde{\delta}_{1} \circ \tilde{\delta}_{0}^{\tau_{3}-\tau_{2}-1} \ldots \ldots \tilde{\delta}_{1} \circ \tilde{\delta}_{0}^{t-\tau_{k} h}\right|_{x_{0}} \cdot$

the following notation has been used for a compact expression of the kernels (here $\delta_{0}^{-1}$ has not been defined and carries no meaning)

$$
\tilde{\delta}_{i_{1}} \circ \tilde{\delta}_{0}^{-1} \circ \tilde{\delta}_{i_{2}} \circ \tilde{\delta}_{0}^{-1} \circ \ldots \circ \delta_{0}^{-1} \circ \tilde{\delta}_{i_{r}}=\tilde{\delta}_{r}, \quad r \geqslant 0, \quad i_{1}=\ldots=i_{r}=1 .
$$

We get the kernels for the system (6), (2) if we replace « in (8) by $« * »$.

Let us now study the Volterra kernels of the closed-loop systems (5), (2) and (6), (2). From (8) it is not difficult to observe that $k$ th kernel $w_{k}$ of the system (5) (2) will depend on $\tilde{\delta}_{1}, \tilde{\delta}_{2} \ldots, \tilde{\delta}_{k}$ and not upon $\tilde{\delta}_{m}, m>k$. Moreover, $\tilde{\delta}_{s}$ in turn will depend on $\xi_{1}, \xi_{2} \ldots, \xi_{s}$, and not on $\varepsilon_{l}, l>S$ (see (7)). Therefore, the $k$ th kernel $w_{k}$ will depend on $\varepsilon_{1}, \ldots, \varepsilon_{h}$, and not on $\varepsilon_{l}, l>k$. Analogous observations can be made for the system (6), (2): the $k$ th kernel will depend on $\gamma_{1}, \ldots, \gamma_{k}$, and not on $\gamma_{l}, l>k$. As $\xi_{i}=\gamma_{i}$ for $i \leqslant r$. it follows that the kernels up to $k$ th one will coincide for systems (5), (2) and (6), (2).

\section{REFERENCES}

1. Котта Ю. // Кнбепнетика и вычислительная техника. 1989 (в печати)

2. Monaco, S.. Normand-Curot. D. // Int. J. Control.. 1983. 38. № 1. 245-261.

3. Monaco, S. Normand-Cyrot, D. // Proc. 22nd IEEE Conf. Decis. and Control., 2. San Antonio. 1983, 655-660.

4. Normand-Cyrot, D. Théorie et pratique des systems non linéaires en temps discret. Thèse le grade de Docteur d'etet. Université de Paris-Sud, 1983.

5. Прудников А. П., Брочков Ю. А., Маричев И. Интегралы и ряды. М., Наука, 1986.

Academy of Sciences of the Estonian SSR,

Received Institute of Cybernetics

Feb, 28, 1989 